\title{
COMPARAISON ET EXPRESSION DU HAUT DEGRE DANS LE GROUPE NOMINAL
}

Pierre-André Buvet \& Gaston Gross

Laboratoire de Linguistique Informatique

Université PARIS XIII-INALF

1. Vraies et "fausses" comparatives

Kr. Sandfeld distingue parmi les comparatives "celles qui marquent un rapport d'égalité ou d'inégalité d'une action ou d'un fait quelconque avec une autre action ou un autre fait." (1965 p. 524). Dans les deux cas, il s'agit de la mise en parallèle d'une propriété de deux sujets par l'intermédiaire de leurs prédicats, que ceux-ci aient le statut/ la forme\$ morphologique d'un adjectif, d'un verbe ou d'un adverbe. La comparaison est "ouverte", dans la mesure où c'est le locuteur qui établit la comparaison, c'est-à-dire qui pose une prédication. Ceci a pour conséquence que la détermination du second élément n'est pas contrainte.

Cependant, il existe des constructions figées : certains adjectifs suivis du groupe comme $\mathrm{N}$ n'ont pas une interprétation comparative mais expriment l'intensité ou le haut degré :

Luc est fort comme un Turc

Ce drap est blanc comme neige

Maurice Gross 1986 a étudié en détail ces constructions adjectivales et en a donné une liste quasi exhaustive.

L'utilisation de la forme comparative pour exprimer le haut degré se rencontre aussi dans des groupes nominaux du type $\mathrm{N}$ de $\mathrm{N}$ où la suite de $\mathrm{N}$ exprime une comparaison qui n'indique pas "une qualité égale, supérieure ou inférieure à la même qualité considérée dans un autre être ou un autre objet" (M. Grevisse 1986, p. 391) mais traduit l'intensité :

Luc a une fièvre de cheval $=$ Luc a beaucoup de fièvre 
Nous sommes donc en présence de "fausses comparatives" où l'expression du haut degré repose sur l'association de la propriété considérée avec un terme pris comme le parangon ou le stéréotype de cette propriété.

2. Groupes nominaux à complément intensif en de $\mathrm{N}$

Dans l'optique d'une description exhaustive des structures du type $\mathrm{N}$ de $\mathrm{N}$ entreprise au LLI, nous avons constitué une liste de plus de 400 constructions du type fièvre de cheval. Dans un premier temps, nous présenterons les critères requis pour l'élaboration de ce corpus. Nous examinerons ensuite d'autres aspects de la relation entre $\mathrm{N} 1$ et $\mathrm{N} 2$.

2.1. Propriétés définitionnelles

Deux critères caractérisent les expressions que nous avons répertoriées :

- la suite nominale est une structure partiellement figée ;

- le groupe de $\mathrm{N}$ a une interprétation d'intensif.

\subsubsection{Figement partiel}

Les groupes nominaux peuvent avoir des degrés de figement variés (G. Gross, 1992). Ceux que nous étudions ne sont affectés qu'au titre du complément de $\mathrm{N}$ : le premier substantif, lui, entretient avec le reste de la phrase des relations syntaxiques libres. Les suites du type fièvre de cheval sont donc moins figées que celles du type pomme de terre, dans la mesure où les premières, contrairement aux secondes, autorisent l'effacement du complément de nom, c'est-à-dire un changement de détermination :

Luc est cloué au lit par une fièvre de cheval Luc est cloué au lit par (la + une forte) fièvre

Luc a mangé des pommes de terre \#Luc a mangé des pommes

Le "génitif intensif" est caractérisé par les restrictions suivantes :

- pronominalisation

La possibilité de remplacer N1 ou N2 par un pronom s'avère impossible : Luc n'apprécie pas l'agitation et particulièrement l'agitation de ruche qui règne ici *Luc n'apprécie pas l'agitation et particulièrement celle de ruche qui règne ici Léa aime les épaules d'Hercule (= les épaules larges)

\#Léa aime les épaules de celui-ci

- insertion d'un modifieur

En règle générale, la possibilité d'introduire un élément dans une construction libre du type $\mathrm{N}$ de $\mathrm{N}$ s'observe aussi bien pour le premier substantif que pour le second : Luc mange le gâteau ( $\mathrm{E}+$ délicieux) de ce boulanger Luc mange le gâteau de ce ( $\mathrm{E}+$ charmant) boulanger Ici, l'adjonction d'un modifieur à N2 est interdite : Sa peau est d'une blancheur de $(\mathrm{E}+*$ *bon) lait 
- coordination

Dans une séquence $\mathrm{N}$ de $\mathrm{N}$ non figée, on peut coordonner des compléments de nom à condition que les deux noms aient les mêmes caractéristiques syntaxiques et sémantiques :

Luc observait l'élévation du monte-charge

Luc observait l'élévation de l' ascenseur

Luc observait l'élévation du monte-charge et de l'ascenseur

Cette possibilité est interdite pour les suites à modifieur intensif :

Luc évoquait sa prudence de serpent

Luc évoquait sa prudence de chat

*Luc évoquait sa prudence de serpent et de chat

D'autres faits étayent ce figement. Dans un groupe libre on observe une certaine variation de la détermination comparée des deux substantifs :

Luc ne s'attendait pas à (une + cette) réponse de $(\mathrm{un}+\mathrm{le}+\mathrm{ce}+$ notre $+* \mathrm{E})$

ministre

Les possibilités de variation sont plus limités pour les expressions de haut degré, :

Luc ne s'attendait pas à $($ un + ce $)$ froid de $(E+* u n+* l e+* c e+*$ notre $)$ canard

Dans certains cas (Cf. Supra), on observe :

Luc a un bon sens de E paysan

Luc a le bon sens de un paysan

Le figement de de N2 est également attesté par la quasi-impossibilité de le remplacer par un synonyme :

Il fait un froid de (canard $+*$ colvert $+*$ eider)

Luc nous a imposé une discipline de (fer $+*$ métal)

S'il est vrai que dans certaines suites intensives, on peut remplacer le groupe prépositionnel par une forme adjectivale associée au substantif :

Le mur est d'une blancheur (de lait + laiteuse)

Luc a une force (d'Hercule + herculéenne)

cette possibilité n'est pas cependant la règle :

Luc a une fièvre (de cheval $+*$ chevaline)

Luc a une volonté (de fer $+*$ ferreuse)

De plus, la substitution par un adjectif peut varier en fonction de N1 :

Il y avait un bruit (d'enfer + infernal)

Luc avait une trouille (d'enfer $+*$ infernale)

Les propriétés que nous venons de passer brièvement en revue font apparaître deux caractéristiques des expressions nominales du haut degré :

- d'une part, N1 est le substantif-tête du groupe nominal (i.e. les restrictions de sélection entre ce substantif et le reste de la phrase ne sont pas modifiées par 
le substantif "intensif" ; Cf. N. Chomsky, 1971) ; N2 un élément de sa détermination, un modifieur ;

- d'autre part, la relation entre N1 et N2 est contrainte dans la mesure où elle n'est susceptible d'aucune transformation, la réduction exceptée.

2.1.2. Valeur intensive

Pour établir la valeur intensive des compléments de N2, nous aurons recours à des paraphrases. Nous verrons d'abord les cas les plus évidents puis nous examinerons des situations où la notion d'intensité est plus difficile à définir.

On peut décrire les expressions de haut degré à l'aide d'un indicateur d'intensité forte comme très (Cf. M. Arrivé \& alii 1986, p.214) :

Luc a une faim de loup

Luc a très faim

Comme peu de constructions nominales acceptent cet adverbe, c'est par

l'intermédiaire de la construction adjectivale qu'on attribuera la valeur intensive de très à un groupe prépositionnel de N2 :

Luc a une carrure de malabar

Luc est très carré

Les prédicats sont identiques dans les deux cas ; seul leur statut/forme\$

morphologique diffère. C'est cette identité qui autorise un rapprochement entre la séquence de malabar et l'adverbe très. Pour rendre compte de la valeur intensive des suites nominales, on peut, dans d'autres cas, établir un parallèle entre UN-de $\mathrm{N} 2$ et le déterminant beaucoup de :

Luc a une mémoire d'éléphant

Luc a beaucoup de mémoire

Les descriptions proposées ci-dessus rendent compte de près de $90 \%$ des expressions du corpus. Pour analyser le reste, on peut souvent utiliser des paraphrases comportant des termes comme grand, long, large, gros, etc (Cf. E. Benveniste 1974, p. 197). Ces adjectifs fonctionnent généralement comme des indicateurs d'intensité forte :

Luc a un cou de girafe Luc a un long cou

Il existe également un nombre limité de suites qui expriment une intensité faible.

On peut les décrire comme précédemment. Seule la nature des indicateurs d'intensité associés change :

Luc a un appétit d'oiseau

Luc a peu d'appétit

Luc vit avec un salaire de misère

Luc vit avec un petit salaire

2.2. Expression du haut degré et métaphore 
Les suites intensives sont une source potentielle de créations lexicales. Nous indiquerons ici quels modèles sont productifs parmi ces expressions nominales. Nous nous limiterons aux cas où N1 est un substantif prédicatif.

Pour interpréter une séquence $\mathrm{N}$ de $\mathrm{N}$ comme une expression du haut degré, il faut tenir compte des caractéristiques de la structure interne du groupe nominal. Nous distinguerons deux types de suites $\mathrm{N} 1$ de $\mathrm{N} 2$ en fonction de la nature de la relation entre les deux substantifs.

La plupart des expressions que nous avons répertoriées sont ambiguës ; on peut les interpréter de deux façons, comme dans :

L'enthousiasme de débutant de Luc fait plaisir à voir

où la séquence enthousiasme de débutant ne traduit la haut degré que si l'attribut\$ débutant ne réfère pas à Luc. Dans le cas contraire, le groupe nominal a un sens compositionnel dont la source est :

Luc est un débutant qui a de l'enthousiasme

La cohérence de la relation entre $\mathrm{N} 1$ et $\mathrm{N} 2$ du point de vue distributionnel explique cette particularité de nombreuses suites du corpus. A côté de suites possibles comme :

Luc a une patience d'ange

Luc a la patience d'un ange on note l'impossibilité de :

Luc a une santé de fer *Luc a la santé du fer où l'on relève une "anomalie sélectionnelle" (Cf. M. Prandi 1987, p. 144-149). Nous partirons de ces observations pour répartir les items du corpus autour de deux pôles.

La première catégorie comporte des suites du type patience d'ange telles que :

- la relation entre $\mathrm{N} 1$ et $\mathrm{N} 2$ ne déroge pas aux règles distributionnelles ;

- l'adjonction de N2 à N1 est pertinente pour définir cette propriété d'une façon hyperbolique ; cela suppose notamment que le second substantif soit nettement différencié de l'argument sujet du premier substantif. Ce type d'expression du haut degré apparaît comme le résultat d'une sélection : celle d'un terme parmi les arguments d'un prédicat donné avec, comme critère de sélection, la nécessité pour ce terme d'être représentatif du caractère extrême de la propriété désignée par le substantif prédicatif. On peut parler ici de "comparaison stéréotypée" (J. Dubois \& alii 1970, p. 111).

Dans la deuxième catégorie, on trouve des séquences où l'indication d'une intensité s'exprime différemment : N2 ne figure jamais dans le domaine d'arguments du prédicat N1. L'association de ces deux substantifs fonctionne à partir d'un trope : 
une synecdoque particularisante dans la suite santé de fer. La séparation entre ces deux catégories n'est pas absolue. Ainsi, dans :

Ce texte est d'une limpidité de cristal

le terme limpidité ne désigne pas la même qualité selon qu'il se rapporte à texte ou à cristal ; il y a donc métaphore. Néanmoins, c'est sur la base d'une comparaison stéréotypée que l'on interprète cette suite comme une expression du haut degré.

Des suites comme fièvre de cheval ou mal de chien sont des cas intermédiaires. Si l'interprétation de ces suites ne relèvent pas de la rhétorique, l'association entre N1 et N2 se laisse difficilement analyser, d'un point de vue synchronique, comme le résultat d'une sélection. Nous concluons en constatant que c'est surtout dans des suites du type patience d'ange que l'on trouve des créations lexicales comme l'attestent ces exemples relevés dans la presse :

Ils se sont défendus avec une énergie de légionnaire (Le Canard Enchaîné 11/5/94)

Ce bagout de bateleur qui avait tant déplu à ses examinateurs (Le Monde 30/06/94)

Naîtront-ils avec des mollets d'athlète et un souffle de plongeur? (Le Monde 29/06/94) 


\section{REFERENCES}

Arrivé M., Gadet F. et Galmiche M., 1986, La grammaire d'aujourd'hui, Paris, Flammarion.

Benveniste E., 1974, Problèmes de linguistique générale 2, Paris, Gallimard.

Chomsky N., 1971, Aspects de la théorie syntaxique, Le Seuil, Paris.

Dubois J., Edeline F.,Klinkenberg J. M., Minguet P., Pire F.et Trinon H., 1970, Rhétorique générale, Paris, Larousse.

Grevisse M., 1980, Le bon usage, Paris-Gembloux, Duculot.

Gross G. 1992, Syntaxe du complément de nom, Linguisticae Investigationes

XV:2, Amsterdam, John Benjamin's B.V..

Gross M., 1986, Grammaire transformationnelle du français Syntaxe de l'adverbe, Paris, ASSTRIL.

Prandi, M. 1987, Sémantique du contresens, Paris, Editions de Minuit.

Sandfeld, Kr.1965, Syntaxe du français contemporain Les propositions subordonnées, Genève, Droz. 